\title{
4D Digital Subtraction Angiography: Implementation and Demonstration of Feasibility
}

\author{
B. Davis, K. Royalty, M. Kowarschik, C. Rohkohl, E. Oberstar, B. Aagaard-Kienitz, D. Niemann, O. Ozkan, C. Strother, and C. Mistretta
}

\begin{abstract}
BACKGROUND AND PURPOSE: Conventional 3D-DSA volumes are reconstructed from a series of projections containing temporal information. It was our purpose to develop a technique which would generate fully time-resolved 3D-DSA vascular volumes having better spatial and temporal resolution than that which is available with CT or MR angiography.
\end{abstract}

MATERIALS AND METHODS: After a single contrast injection, projections from the mask and fill rotation are subtracted to create a series of vascular projections. With the use of these projections, a conventional conebeam CT reconstruction is generated (conventional 3D-DSA). This is used to constrain the reconstruction of individual 3D temporal volumes, which incorporate temporal information from the acquired projections (4D-DSA).

RESULTS: Typically, 30 temporal volumes per second are generated with the use of currently available flat detector systems, a factor of $\sim 200$ increase over that achievable with the use of multiple gantry rotations. Dynamic displays of the reconstructed volumes are viewable from any angle. Good results have been obtained by using both intra-arterial and intravenous injections.

CONCLUSIONS: It is feasible to generate time-resolved 3D-DSA vascular volumes with the use of commercially available flat detector angiographic systems and clinically practical injection protocols. The spatial resolution and signal-to-noise ratio of the time frames are largely determined by that of the conventional 3D-DSA constraining image and not by that of the projections used to generate the 3D reconstruction. The spatial resolution and temporal resolution exceed that of CTA and MRA, and the small vessel contrast is increased relative to that of conventional 2D-DSA due to the use of maximum intensity projections.

ABBREVIATIONS: $\|$ = image intensifier; IA = intra-arterial; TOA = time of arrival

n 1980, when DSA was introduced, ${ }^{1-3}$ it was originally hoped that intravenous $\mathrm{x}$-ray angiography would be feasible. However, the inability to clearly visualize vascular structures caused by vessel overlap as well as limited signal-to-noise ratio due to then available contrast medium and the image intensifier (II) detector technology quickly led to the adaption of techniques by use of small intra-arterial (IA) contrast injections in combination with the newly available real-time digital imaging capabilities. This capability greatly facilitated the development of the minimally invasive procedures by using $\mathrm{x}$-ray angiography and the field of interventional radiology in general.

Received August 29, 2012; accepted after revision December 21.

From the University of Wisconsin School of Medicine and Public Health (B.D., E.O., B.A.-K., D.N, O.O., C.S., C.M.), Madison, Wisconsin; Siemens AG (K.R.), Healthcare Sector, Hoffman Estates, Illinois; and Siemens (M.K., C.R.), Research Collaborations, AX, Forchheim, Germany.

Please address correspondence to Charles Strother, MD, Department of Radiology, University of Wisconsin School of Medicine and Public Health, 600 North Highland Ave, Madison, WI 53792; e-mail: cstrother@uwhealth.org.

http://dx.doi.org/10.3174/ajnr.A3529
The feasibility of obtaining rotational volume acquisitions by using a C-arm angiographic system equipped with an II was initially simulated in 1988 and then by use of an II-based apparatus, implemented in 1996 by Ning and Kruger. ${ }^{4,5}$ In 1997, Fahrig et al ${ }^{6,7}$ extended this work showing both in vitro and in vivo results. These systems, as well as currently used systems with flat detectors, allow creation of $13 \mathrm{D}$-DSA volume per gantry rotation. Since the introduction of commercial angiographic systems and software suitable for performing 3D-DSA in 1999, the assessment of vasculature in interventional $\mathrm{x}$-ray suites has been done by using the combination of 3D-DSA volume reconstructions (non-time-resolved) and multiple conventional 2D-DSA acquisitions. Multiple 2D acquisitions have been necessary because of 1) the need to visualize the sequences of vascular filling and 2) the inability to clearly see anatomic details on the $3 \mathrm{D}$ reconstructions because of the resulting overlap of arterial and, in some instances, venous structures. The need to obtain multiple $2 \mathrm{D}$ temporal series at the desired view angle obviously contributes significantly to the radiation exposure and contrast medium dose associated with these examinations. 


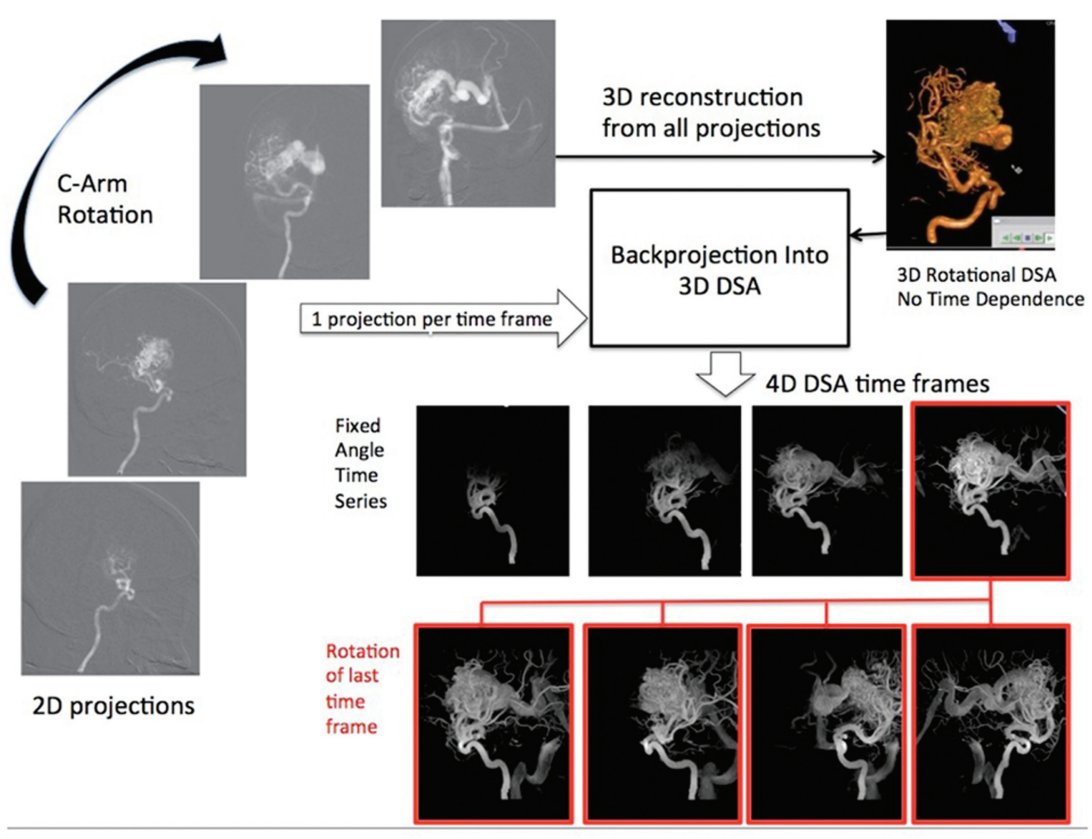

FIG 1. Schematic of 4D-DSA reconstruction. After creation of the 4D-DSA timeframes, each one may be viewed in a dynamic form showing the inflow and outflow of contrast from the vasculature, just as with a standard 2D-DSA series. As shown on the 2 series of 4 images at the bottom of the illustration, any selected time (in this case, the image framed in red in the top row of 4 images) may be viewed at any desired angle. Four possible views of the selected image are shown in the bottom row of 4 images.

The method that we describe involves the use of a 3D-DSA vascular volume as a constraining image. The proposed reconstruction method uses all, or most of, the projections obtained after a rotational acquisition done before (mask run) and after (fill run) the injection of contrast medium. This constraining volume is combined with the acquired projections to form a time-series of $3 \mathrm{D}$ volumes (4D-DSA). The 3D-DSA depicts an average of the vascular opacification present during the rotational acquisition. Each of the individual projections used to form the standard 3DDSA volume contains temporal information. Although it must be understood that a CT volume cannot be reconstructed from a single projection, we do not propose a new 3D-DSA volume reconstruction technique. We have developed algorithms that permit 3D-DSA time-resolved volumes (4D-DSA) to be generated by use of the constraining volume and single $\mathrm{x}$-ray projections. Rather than 1 vascular volume per gantry rotation, this technique typically generates 30 time-resolved 3D-DSA volumes per second (by use of a currently available flat detector angiographic system), a rate $\sim 200$ times faster than could be achieved by use of sequential gantry rotations, and at the same contrast and $\mathrm{x}$-ray dose as used to obtain 1 standard 3D-DSA volume.

This capability allows for the viewing of the vasculature in a dynamic display, at any angle, for all time points at any desired angle. This largely eliminates the problem of vessel overlap. The availability of a "library" of time-resolved 3D timeframes thus facilitates diagnosis by providing the ability to view the anatomy not only in any desired view but also to do this at any desired phase of vascular filling, thus eliminating any confusion that might occur as the result of vascular overlap. An additional benefit is that it provides a library of vascular roadmaps at any stage of vascular filling for use in endovascular therapeutic procedures.
The purpose of the work was to investigate the feasibility of reconstructing time-resolved 3D vascular volumes (4D-DSA) from conventional 3D-DSA acquisitions.

\section{MATERIALS AND METHODS Data Acquisition}

All images were acquired by use of either a biplane Artis dBA system or an Artis Zeego flat detector angiographic system (Siemens, Erlangen, Germany). All human and animal studies were performed under institutionally approved institutional review board and/or Animal Care and Use Committee protocols. Rotational images for subtracted $3 \mathrm{D}$ reconstructions were obtained by use of 5-, 8-, or 10-second acquisitions to capture the full cycle of contrast inflow and washout. To obtain a sufficient number of consistent projections for an accurate $3 \mathrm{D}$ reconstruction and to capture inflow and washout, the rotational acquisition must be long enough to follow a bolus through the vasculature. This may be achieved by use of either multiple C-arm rotations or by use of C-arm rotations of an appropriate duration, for example, 5, 8, or 10 seconds. It seems likely that, depending on the dynamics of the circulation, that is, fast or slow, there will be particular acquisition protocols that are optimal for particular conditions, for example, a 5-second acquisition for evaluation of a patient with an aneurysm at the circle of Willis or a fast-flow carotid cavernous fistula or an 8- or 10-second acquisition for a patient with occlusive disease in which filling occurs by collaterals. More experience is required before definite recommendations can be given regarding choice of an optimal acquisition protocol. Intravenous studies were performed after injection of iohexol $(300 \mathrm{mg} \mathrm{I} / \mathrm{mL})$ into a peripheral vein at the rate of $4 \mathrm{~mL} / \mathrm{s}$ over $8-10$ seconds followed by a $10-\mathrm{mL}$ saline chase injected at the same rate. Intra-arterial studies were obtained by use of injection of 5-8 $\mathrm{mL}$ iohexol.

\section{Image Processing and Reconstruction Process}

Four-dimensional DSA reconstruction uses multiplicative projection processing to generate a series of time-resolved 3D volumes (4D-DSA) by embedding the time-resolved data from the standard projections of a rotational acquisition. Before back projection, the individual projections from the acquisition are spatially convolved to increase the SNR. They therefore form a low spatial frequency mask that basically enhances portions of the 3D constraining image that are present at each point in time. After a normalization step, this mask then provides proper projection weighting. As a result of the convolution process, the SNR of the individual timeframes is limited by the constraining image SNR ratio and not by the SNR of the individual projections. When the single projections are back-projected into the $3 \mathrm{D}$ volume, there is a tendency for the projection values from overlapping vessels to 
acquired projection

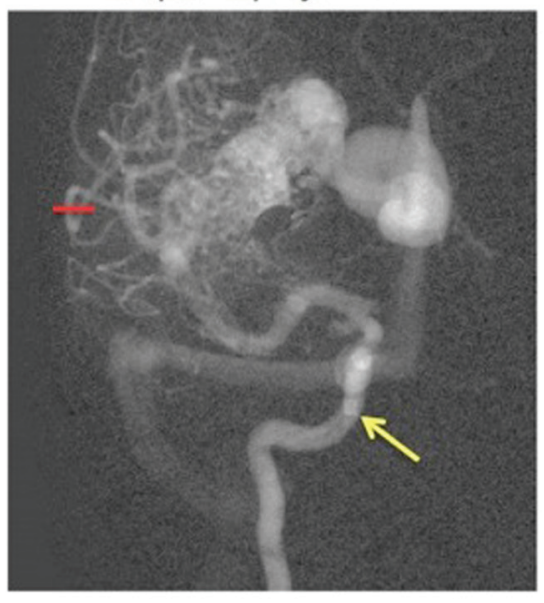

$\operatorname{snr}=8.17$
4D DSA

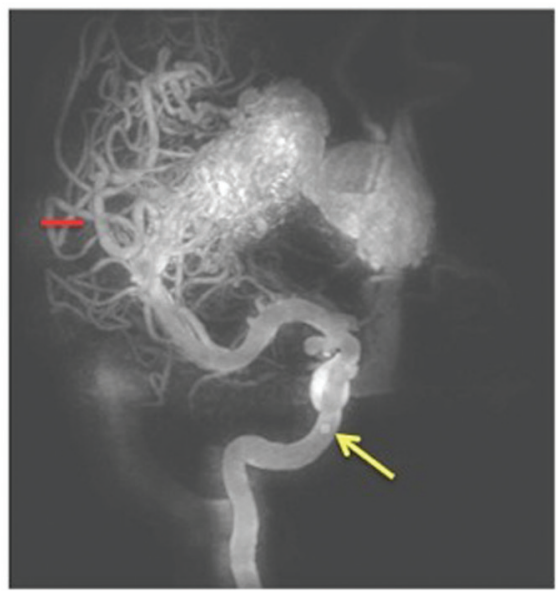

snr $=38.5$
3D DSA

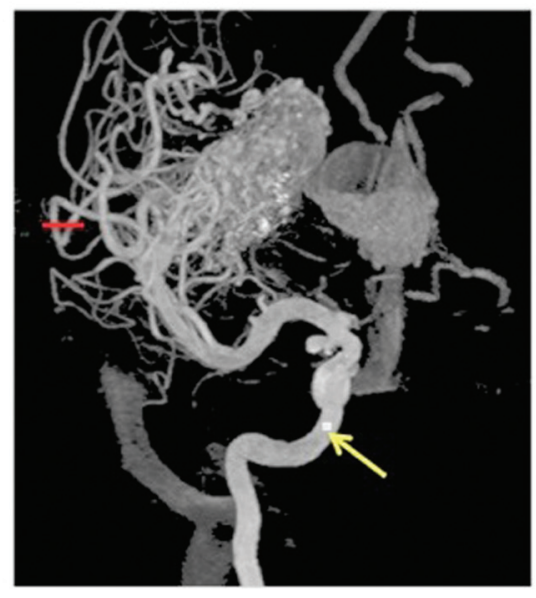

$\operatorname{snr}=42.6$

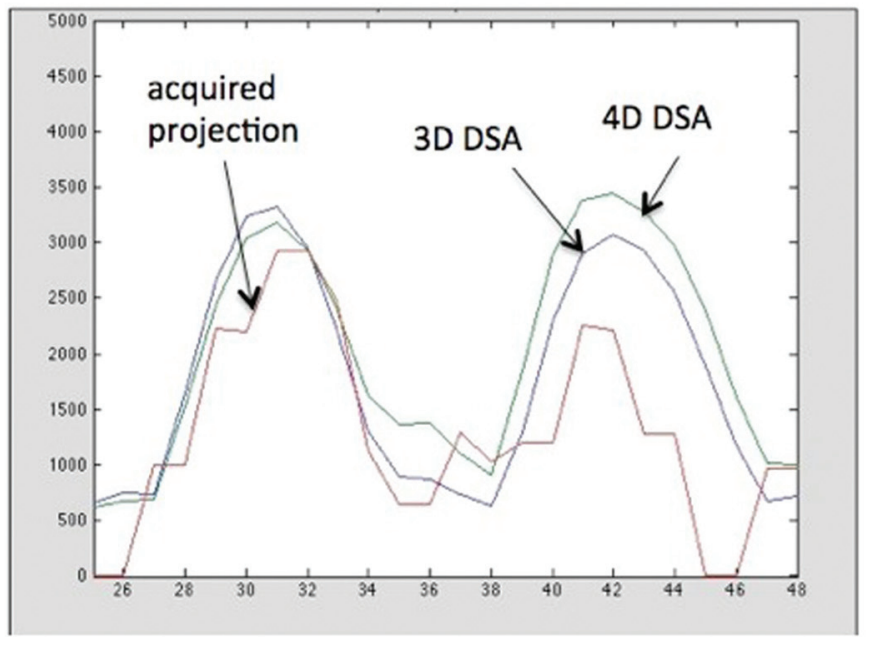

FIG 2. Images show a selected projection from a 3D-DSA rotational acquisition (top left), a 4D-DSA timeframe (center), and a standard 3D-DSA reconstruction (right). The SNRs of the 3 images at the region indicated by the yellow arrows are shown beneath each image. The single image at the bottom shows traces of the profile across the 2 arteries indicated by the red line. These images were obtained by use of an IA injection of contrast medium.

cause the deposition of erroneous signal (an opacity shadow from opacified vessel to nonopacified vessel) into vessels in the $3 \mathrm{D}$ volume. To reduce this effect, for each timeframe, an angular (temporal) search is performed, looking for a range of time before and after the frame that is being projected. After this search, the minimum signal for each voxel is assumed to be due to the ray with the minimum degree of overlap; that value is then assigned to the timeframe being processed. For voxels requiring an angle other than the current angle as a result of the search, the temporal resolution is lower than the typical 30/s rate, as data from a different timeframe are substituted. The frequency of this depends on the sparsity of the vessel configuration. However, overall volume frame rates of 6 frames/s are typical. Figure 1 illustrates the steps in this process. Any of the time-resolved 3D timeframes (3D volumes) can be rotated for viewing at whatever angle the user thinks gives the clearest depiction of the relevant vasculature. These 3D timeframes can be viewed with playback speed reduced or increased, paused, or replayed as desired. The user interface provides for interactive $4 \mathrm{D}$ viewing of the $3 \mathrm{D}$ temporal volume timeframes and provides for a true interactive $4 \mathrm{D}$ viewing experience of the contrast-enhanced perfusion of a vascular network.

\section{Estimate of Relative SNR}

The SNR values for the acquired projections, for 3D-DSA and for $4 \mathrm{D}$-DSA, were estimated by placing regions of interest in the images. Because variations of the signal selected by the maximum intensity projection process can contribute to the standard deviation in a region of interest, the statistical variations in the $4 \mathrm{D}$-DSA image were measured from the difference between 2 successive $4 \mathrm{D}$-DSA frames that were rotated to the same position. In the $3 \mathrm{D}$-DSA, these variations were very small, so no subtraction was performed. It was not possible to subtract sequential acquired projections because they are not $3 \mathrm{D}$-rotatable and cannot be registered for subtraction. Thus, the presence of these potential signal variations in the acquired 


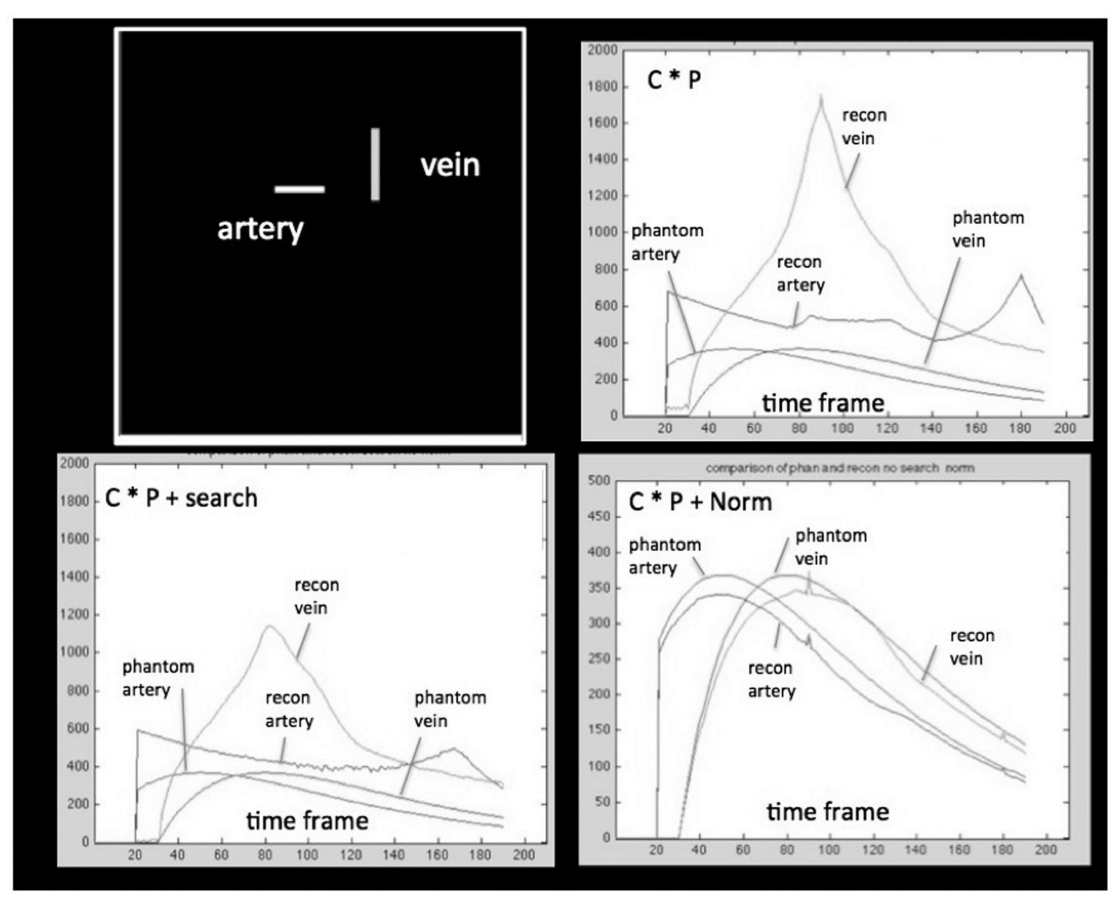

FIG 3. Demonstration of the effects of reconstruction elements on accuracy of reconstructed simulated vessel signal curves. The digital phantom is shown in the top frame on the left. The vessels in the phantom (phantom artery and phantom vein) had specified time dependence. These are considered to represent ground truth. The input time curves for these vessels are shown in each panel for comparison. $C$ indicates constraining image; $\mathrm{P}$, projections. The reconstructed wave forms are shown: 1) after multiplication (represented by $C \times P$ ) of the projections by a binary constraining image (top right); 2) multiplication of the projections by the binary constraining image plus an angular minimum search (represented by C $\times \mathrm{P}+$ search, bottom left); and 3) multiplication of the projections by the binary constraining image followed by normalization by the estimate of the numbers of projected ray voxels obtained from the constraining image $(C \times P+N o r m$, bottom right). The $y$-axis shows arbitrary units of attenuation. The $x$-axis shows the projection number.

projection region of interest could have decreased the measured SNR shown in Fig 2.

\section{Simulation of Flow Information From 4D-DSA}

A digital phantom was constructed to simulate the various steps in signal curve generation. The phantom consisted of a vein and an artery running perpendicular to the vein. Each was assigned venous and arterial $\gamma$-variate time dependences. A 4D-DSA reconstruction was performed with the use of a constraining volume obtained from the reconstruction of the known vessel distribution. Signal curves were then generated from 2 selected regions of interest for different versions of the 4D-DSA reconstruction algorithm.

\section{Quantitative Color-Coded 4D-DSA}

Previous work has shown the ability to create color-coded parametric images from 2D-DSA series. ${ }^{8,9}$ Because the basic 4D-DSA images contain signal that is proportional to the contrast (iodine) concentration as a function of time, it is possible to also view these series as a color-coded display showing contrast time of arrival (TOA) in each pixel. TOA maps were created by first forming a volume showing the highest contrast concentration, that is, vessel opacification that occurred during the rotational acquisition (Cmax) and noting the time to reach $\mathrm{Cmax} / 4$ on a per-voxel basis. Color-coded 4D-DSA images were obtained by multiplying TOA maps by a binarized version of the 4D-DSA MIP timeframes.
A method for displaying regions of vascular anatomy with increasing times of the contrast bolus arrival can be achieved by multiplying the TOA image by a sliding window that sequentially reveals pixels within a TOA range defined by the window. We refer to this as a bolus arrival mode. For the results illustrating this capability, we used a Gaussian display window with full width at half maximum of $204 \mathrm{D}$ DSA timeframes.

\section{RESULTS}

\section{Simulation of Reconstruction Elements}

The angular search results in some distortion of the signal waveforms. When it is desired to generate quantitative signal curves, the effect of this can be limited because it is possible to omit points from the waveform for which the search resulted in the choice of a projection that is too remote (in time) from the current frame. The signal curve then can be interpolated by use of points for which the search resulted in a suitable temporal displacement, for example, below a chosen threshold.

For the purpose of generating signal curves that correspond exactly to the signal curves in the acquired projections, it is possible to modify the basic reconstruction algorithm to include a binary version of the constraining image. In this case, the projection values are directly deposited in the $3 \mathrm{D}$ vascular space and will reproduce the signal curves provided by the projections. This series of $3 \mathrm{D}$ volumes does not accurately represent the actual opacification of the vascular network because of vessel overlap and the use of the back-projection step used to create these volumes. However, for the purpose of generating individual voxel signal curves, there are several characteristics of the 4D-DSA reconstruction algorithm that affect the resultant curves that aim to correct the vessel overlap problem inherent in the $3 \mathrm{D}$ timeframe volumes. This is demonstrated in the simulations performed with the digital phantom.

As shown in Fig 3, after the basic back-projection step when the projections are multiplied by a binary constraining image (upper right), there are signal peaks caused by the alignment of the vessels with the projection direction. When these are present, they can occasionally give the impression of reversed flow. These peaks are somewhat reduced when the angular search is added to the reconstruction (Fig 3, lower left panel). Better results are obtained when the projections are normalized by dividing them by an estimate of the number of aligned voxels along the individual rays, obtained from the constraining image (Fig 3, lower right panel). These normalizing projection images ( 1 per projection) are created by forward projections of the thresholded and binarized con- 

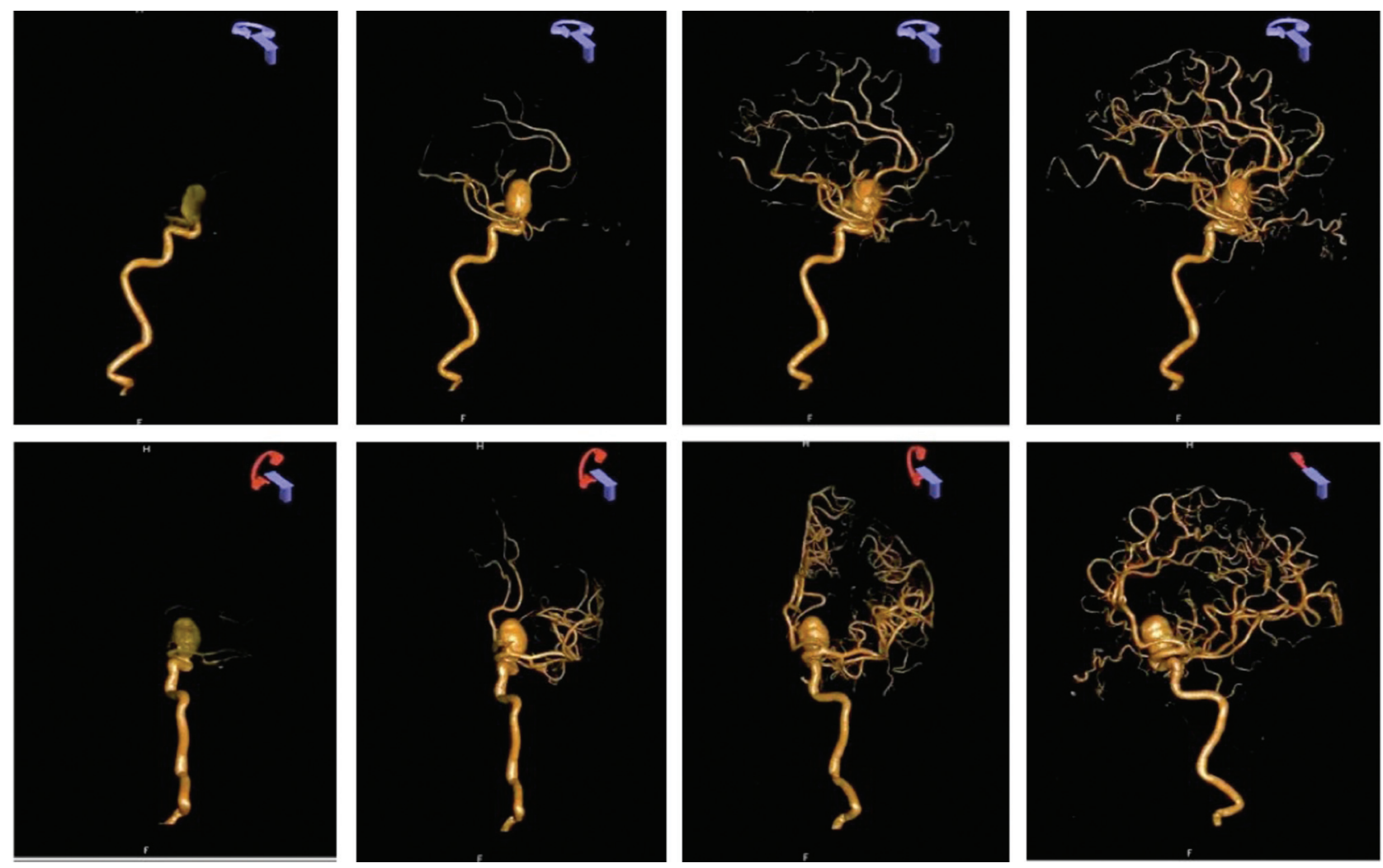

FIG 4. Four-dimensional DSA reconstruction from a 3D-DSA reconstruction performed for evaluation of an unruptured paraclinoid aneurysm. An IA injection of contrast was used for this examination. The top row shows selected timeframes viewed at the rate of 6 frames per second at a fixed viewing angle. The bottom row shows the same timeframes viewed at 4 different angles, which would have not been obtainable in a biplane acquisition because of the mechanical inability to position the A-plane gantry (red icon).

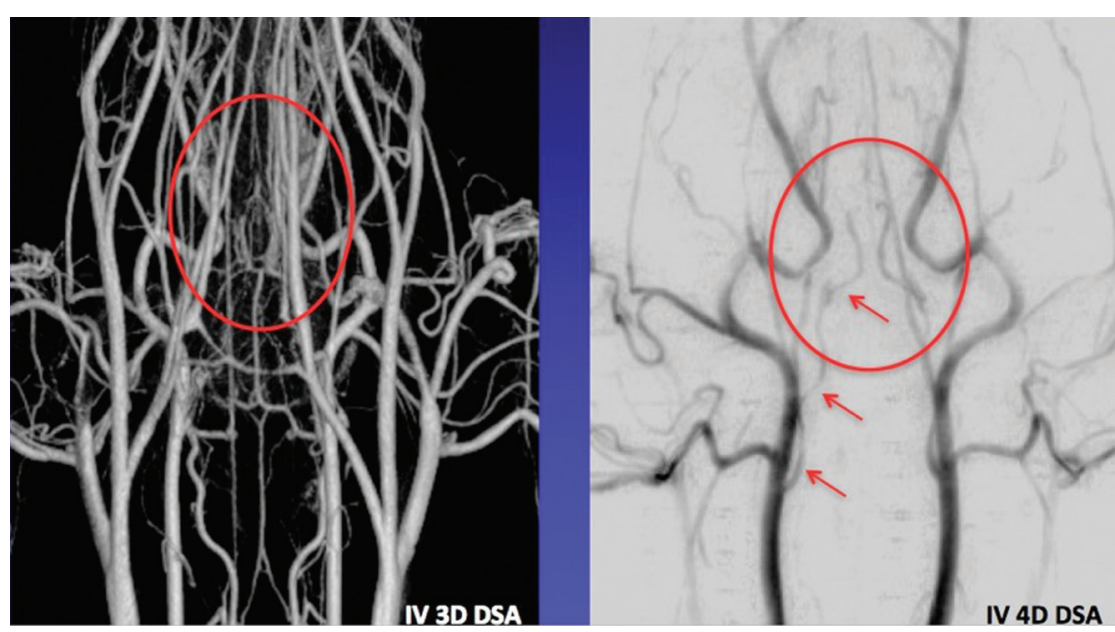

FIG 5. Comparison of intravenous 3D-DSA and early arterial phase time from a 4D-DSA reconstruction. In the standard 3D-DSA on the left, the overlap of both arteries and veins obscures visualization of the internal carotid arteries. In this example, there is no viewing angle that will eliminate this overlap in the 3D-DSA. The image on the right is an early arterial timeframe from a 4D-DSA reconstruction viewed from the same angle as the 3D-DSA on the left. In this 4D image, the full course the right internal carotid artery is clearly visualized (red arrows). The red ellipse shows the position of the distal segments of the internal carotid arteries in both images.

straining image. With the use of this method, the similarity of the reconstructed signal curves to those of the input signal curves is satisfactory. This technique effectively helps to weight deposition of the attenuation coefficient in voxels along a ray, resulting in more accurate 4D-DSA raw 3D temporal volume when a normalized projection is back-projected.

\section{Anatomic Display}

Imparting temporal resolution to an already reconstructed $3 \mathrm{D}$ vascular volume requires far fewer multiplicative projections than if the goal were to reconstruct a vascular (anatomic) volume itself by summing a limited number of projections. In our technique, the anatomic structures in those voxels have already been reconstructed. Time dependence can then be effectively performed with the use of a small number of multiplicative projections. ${ }^{10,11}$

Figure 4 shows a series of $4 \mathrm{D}$-DSA arterial phase images from a study performed for evaluation of an unruptured aneurysm. These illustrate the ability to view the time-resolved $4 \mathrm{D}$ series from any desired viewing angle. This ability to retrospectively view a dynamic angiographic sequence from any arbitrary view angle can potentially result in a significant reduction in radiation exposure because, in our experience, to obtain optimal views, it is not unusual to require multiple $2 \mathrm{D}$ acquisitions even when performing a "tar- 


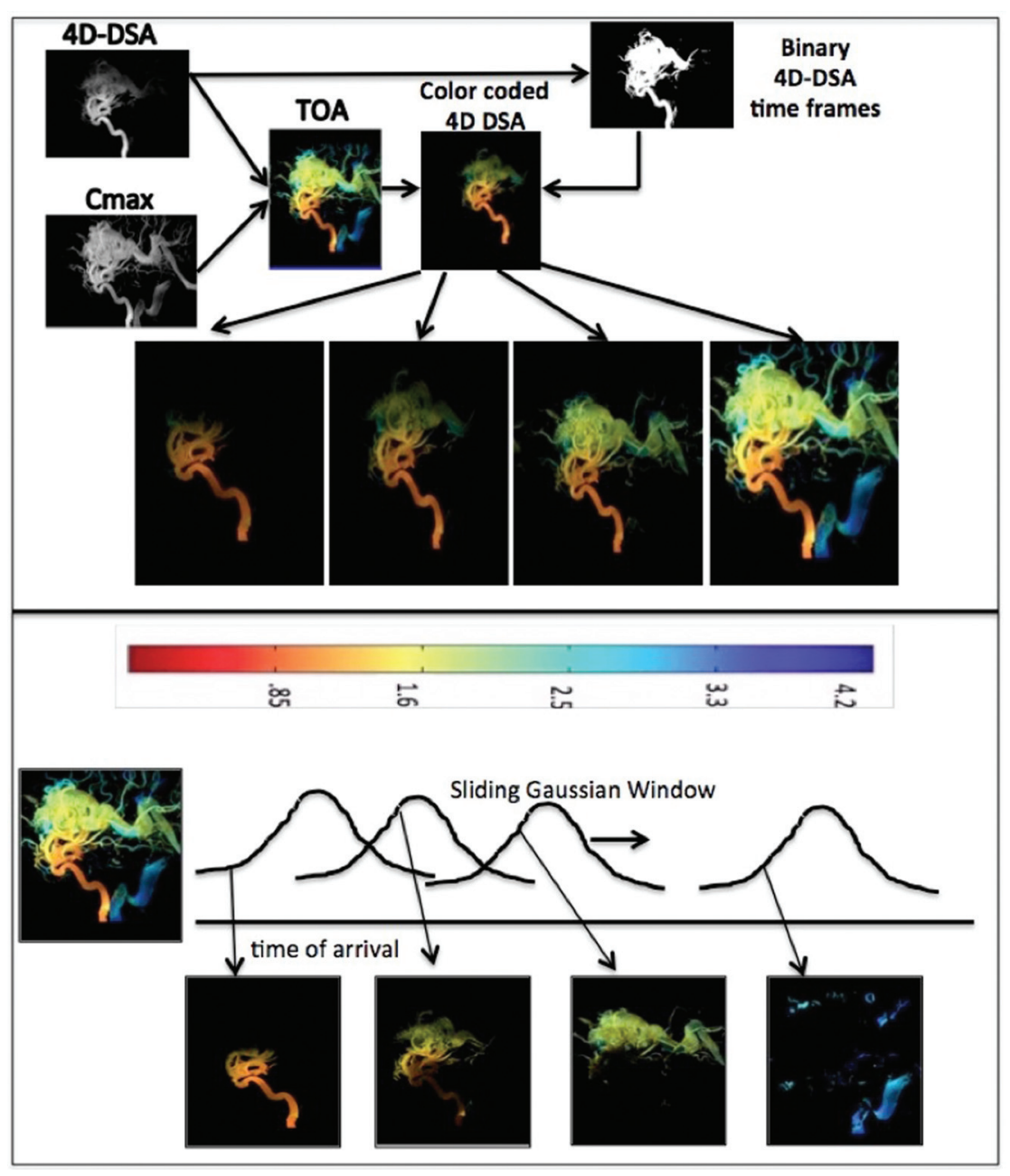

FIG 6. Color-coded 4D-DSA and bolus arrival images. The sequence was displayed at a rate of 6 frames per second for visualization of arrival times between 1 and 4.5 seconds. On the top of the figure, binarized 4D-DSA timeframes multiply a time-of-arrival map providing a dynamic display in which each pixel in each timeframe is represented by a quantitative time of arrival value. In the bottom half of the figure, a sliding Gaussian display window is used to show the passage of the bolus through the AVM. The 4D-DSA TOA volume (static) and 4D-DSA TOA (dynamic) 3D timeframes can be viewed from any angle. The 4D-DSA TOA (dynamic) 3D timeframes allow viewing of the temporal dynamics of the 4D-DSA TOA from any angle at any point in time for which data were acquired. These images were obtained by use of an IA injection of contrast medium.
SNR of the acquired projection may have been decreased because of anatomic variations during gantry rotation. Also shown are normalized vessel profiles along the red lines indicated. The profile across these 2 arteries on the acquired projection is noisy. Determination of the exact 4D-DSA spatial resolution requires more careful experiments with the use of well-defined phantoms. These studies are ongoing.

Figure 6 illustrates the ability to color-code a 4D-DSA image such that quantitative information about blood flow can be extracted. This example shows a $4 \mathrm{D}$ reconstruction of a study performed for evaluation of an AVM.

\section{DISCUSSION}

In this report, we have demonstrated the feasibility of generating time-resolved 3D-DSA vascular volumes with the use of commercially available flat detector angiographic systems and clinically practical injection protocols. A review of other angiographic methods (both timeresolved and non-time-resolved) indicates that $4 \mathrm{D}$-DSA can provide spatial and temporal resolution that exceeds that of these competing techniques, for example, MRA and CTA. In particular, 4D-DSA thus provides a new imaging technique with spatial and temporal resolution exceeding competing time-resolved angiographic techniques. Figure 7 shows the relative voxel volume and frame duration for existing time-resolved angiographic methods. We emphasize that this was a feasibility study with implementation of the technique geted" (single vessel) diagnostic examination. This ability also removes the need to obtain extreme cranial-caudal views that require passing $\mathrm{x}$-rays through a highly attenuating path. With 4D-DSA, such views can be achieved with the use of a reconstruction acquired from data acquired such that rays traverse a much less attenuating trajectory.

Our initial efforts to use intravenous injections of contrast in conjunction with DSA were hampered by the overlap of vascular structures on the 2D images. With 4D-DSA, this limitation is largely overcome because optimal views, which avoid overlap, can be chosen and viewed at any stage of vascular filling (any view angle at any timeframe and any timeframe at any view angle). This is illustrated in Fig 5 , in which the course of the right internal carotid artery can be clearly seen in the early arterial phase 4D-DSA timeframe.

\section{SNR and Vessel Profiles}

Figure 2 shows a comparison of SNR values measured in the regions of interest indicated by the yellow arrows. The apparent with the use of a prototype environment. Studies to understand the trade-offs of various acquisition and injection protocols are underway. Full understanding and optimization of these protocols will require significantly more experience and use of the technology.

Validation studies will be required to establish the reconstructed matrix size and radiation dose that would be required to provide the highest-resolution $4 \mathrm{D}$-DSA volumes. It is interesting to note that despite recent research developments in MR, the dominant commercial method is still time-resolved imaging of contrast kinetics (TRICKS), which has spatial and temporal resolution 2 orders of magnitude below that of 4D-DSA. ${ }^{12}$

The availability of 4D-DSA provides the user with a technique that, from a single contrast injection and rotational acquisition (provided an acceptable constraining volume can be obtained), provides a library of time-resolved images that may be viewed from any desired angle at any time during contrast filling and 


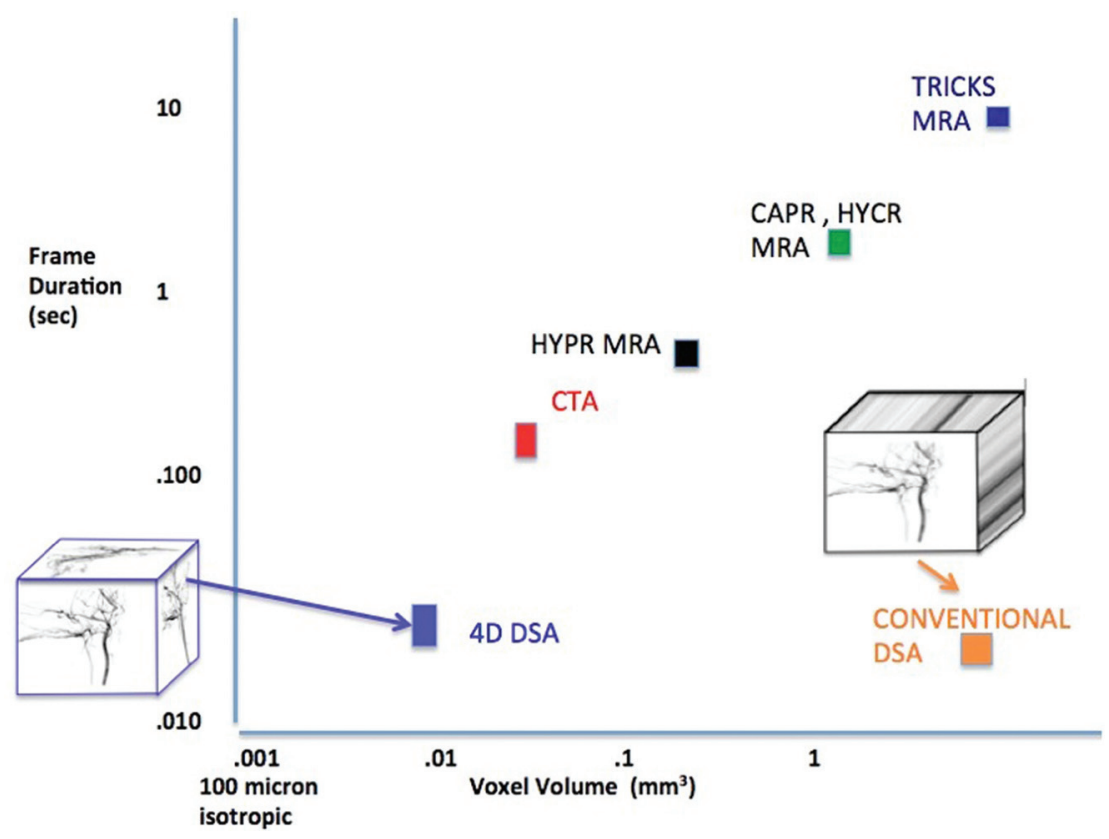

FIG 7. Relative spatial and temporal resolution for competing time-resolved angiography methods. The MRA values are based on the hybrid MRA method reported by Wu et $\mathrm{al}^{12}$ and provide $0.69-\mathrm{mm}$ pixel dimensions, leading to a voxel volume of $0.33 \mathrm{~mm}^{3}$. Typical frame times are 0.3 seconds. The CTA estimate is based on the large area Aquilion system (Toshiba Medical Systems, Tokya, Japan) $)^{14}$ with a pixel dimension of 0.625 and frame rate of approximately $3 / s$. The 4D-DSA ultimate spatial resolution assuming a magnification factor of 1.5 and a pixel dimension 100 should easily support $200-\mu$ pixel dimensions in a patient, leading to a voxel volume of $0.008 \mathrm{~mm}^{3}$.

less than 1 second. Clinical validation of the anatomic and temporal accuracy as well as documentation regarding the value of the technique in reducing procedure times, $\mathrm{x}$-ray exposure, and contrast medium dose will start once the technique is developed to a point at which it may be transferred to a clinical angiographic system.

Four-dimensional DSA will ultimately be used along with 4D fluoroscopy, a technique that will provide real-time fluoroscopic roadmap views from any arbitrary direction without a need for gantry movement. Together, 4D-DSA and 4D fluoroscopy should increase diagnostic capabilities and improve the safety and efficacy of endovascular interventions.

\section{CONCLUSIONS}

It is feasible to generate time-resolved 3D-DSA vascular volumes with the use of commercially available flat detector angiographic systems and clinically practical injection protocols. The spatial resolution and SNR of the timeframes are largely determined by that of the conventional 3D-DSA constraining im-

emptying. Although conventional 3D-DSA reconstructions are viewable at any angle, they do not allow viewing of the vasculature during different stages of filling and emptying, for example, a pure arterial phase or pure venous phase. Because 4D-DSA allows this, it should, in our opinion, allow the performance of many procedures requiring the use of $x$-ray angiography with significantly less $\mathrm{x}$-ray exposure and contrast medium dose because it would reduce the need to obtain multiple $2 \mathrm{D}$-DSA acquisitions. We also believe that because the time-resolved volume viewable from any angle at any time will largely eliminate the impact of vascular overlap, that $4 \mathrm{D}$-DSA will provide a method that will again make the use of intravenous injections for angiographic studies an attractive alternative to conventional IA studies, thus potentially increasing the safety (less invasive) and efficacy (obtaining images of all relevant vasculature with a single injection and acquisition) of interventional and diagnostic procedures.

It should be emphasized that 4D-DSA is a purely angiographic technique and as such only indirectly provides perfusion information, that is, it provides information about blood flow to tissue. This is because it is not possible to reconstruct the tissue surrounding vessels from only a single projection. Although the constraining volume is reconstructed from a large number of projections, the tissue perfusion represented by that volume is an average, over time, and thus cannot provide dynamic perfusion information. It is possible, however, that $4 \mathrm{D}$-DSA could provide valuable arterial input function information for alternate schemes of measuring perfusion with a C-arm angiographic system. ${ }^{13}$

All of the images shown in this report were reconstructed retrospectively and were not available at the time of the actual examinations. The reconstruction time per 4D-DSA frame is presently age and not by that of the projections used to generate the $3 \mathrm{D}$ reconstruction. The voxel volume and temporal resolution are theoretically almost 10 times that of CTA and MRA, and the small-vessel contrast is increased relative to that of conventional 2D-DSA as the result of the ability to use MIP renderings. The availability of 4D-DSA should greatly reduce the need to acquire multiple $2 \mathrm{D}$ series during examinations. The availability of $4 \mathrm{D}$ DSA volumes viewable from any angle at any time completes the transition of DSA to a full 4D angiographic technique. ${ }^{12,13}$

\section{ACKNOWLEDGMENTS}

We dedicate this work to the memory of our colleague Dr Jan Bose, who recently passed away. Although Jan was not an author of this manuscript, his insights and guidance were invaluable as we worked to bring this technology from a concept to a reality. Jan was a dear friend and colleague; his memory will never leave us.

Disclosures: Brian Davis—RELATED: Grant: Siemens Medical Sponsored Research Grant;* Support for Travel to Meetings for the Study or Other Purposes: Siemens Medical Sponsored Research Grant; * OTHER RELATIONSHIPS: We have a sponsored research agreement between UW Madison and Siemens Medical. Kevin Royalty—RELATED: Grant: Siemens Medical Solutions, USA, ${ }^{*}$ Comments: Project was at least partially funded by Siemens Medical Solutions, USA; UNRELATED: Employment: Siemens Medical Solutions, USA, Comments: Full-time employee of Siemens Medical Solutions, USA; OTHER RELATIONSHIPS: I am also a graduate student at the University of Wisconsin (Department of BioMedical Engineering). Markus Kowarschik-UNRELATED: Employment: Full-time employee at Siemens Healthcare, Comments: Part of this research was funded by Siemens Healthcare. Erick Oberstar-RELATED: Grant: Siemen's Medical Sponsored Research Grant; ${ }^{*}$ Support for Travel to Meetings for the Study or Other Purposes: Siemens Medical Sponsored Research Grant; ${ }^{*}$ OTHER RELATIONSHIPS: We have a sponsored research agreement between UW Madison and Siemens 
Medical. David Niemann—RELATED: Siemens Healthcare, ${ }^{*}$ Comments: Master Research Agreement between University of Wisconsin and Siemens Health Care. Charles Strother-RELATED: Grant: Siemens Healthcare* Comments: Sponsored research agreement between Siemens HealthCare and UW Madison; Support for Travel to Meetings for the Study or Other Purposes: Siemens Healthcare* Comments: Money for travel to scientific meetings included in budget of research grant; OTHER: License of Intellectual Property; UNRELATED: Consultancy: Siemens Healthcare, Comments: I am an unpaid advisor; Grants/Grants Pending: Siemens Healthcare;* Patents (planned, pending or issued): Siemens Healthcare, Comments: License of IP. Charles Mistretta—RELATED: Grant: Siemens Imaging Solutions, ${ }^{*}$ Comments: Siemens/UW sponsored research grant; Support for Travel to Meetings for the Study or Other Purposes: The Siemens grant provides some travel money; UNRELATED: Patents (planned, pending or issued): Siemens has been licensed to develop 4D-DSA ( ${ }^{*}$ money paid to institution).

\section{REFERENCES}

1. Mistretta CA, Kruger RA, Houk TL, et al. Computerized fluoroscopy techniques for noninvasive cardiovascular imaging: SPIE. Appl Opt Instr Med 1978;152:65-71

2. Kruger RA, Mistretta CA, Houk TL, et al. Computerized fluoroscopy in real time for noninvasive visualization of the cardiovascular system: preliminary studies. Radiology 1979;130:49-57

3. Strother CM, Sackett JF, Crummy AB, et al. Clinical applications of computerized fluoroscopy: the extracranial carotid arteries. Radiology 1980;136:781-83

4. Ning R, Kruger RA. Computer simulation of image intensifierbased computed tomography detector: vascular application. Med Phys 1988;15:188-92

5. Ning R, Kruger RA. Image intensifier-based computed tomography volume scanner for angiography. Acad Radiol 1996;3:344-50

6. Fahrig R, Fox AJ, Lownie S, et al. Use of a C-arm system to generate true three-dimensional computed rotational angiograms: prelimi- nary in vitro and in vivo results. AJNR Am J Neuroradiol 1997;18:1507-14

7. Fahrig R, Moreau M, Holdsworth DW. Three-dimensional computed tomographic reconstruction using a C-arm mounted XRII: correction of image intensifier distortion. Med Phys 1997;24: 1097-106

8. Strother CM, Bender F, Deuerling-Zheng Y, et al. Parametric color coding of digital subtraction angiography. AJNR Am J Neuroradiol 2010;31:919-24

9. Lin CJ, Hung SC, Guo WY, et al. Monitoring peri-therapeutic cerebral circulation time: a feasibility study using color-coded quantitative DSA in patients with steno-occlusive arterial disease. AJNR Am J Neuroradiol 2012;33:1685-90

10. Mistretta CA, Oberstar E, Davis B, et al. 4D-DSA and 4D fluoroscopy: preliminary implementation. Proceedings of SPIE Conference on Medical Imaging. San Diego, California, February 2010

11. Davis B, Kowarschik M, Royalty K, et al. Progress in 4D digital subtraction angiography implementation: technical aspects. Presented at Annual Meeting of the American Society of Neuroradiology, New York. April 23-26, 2012

12. Wu Y, Johnson K, Kecskemeti SR, et al. Time resolved contrast enhanced intracranial MRA using a single-dose delivered as sequential injections and highly constrained projection reconstruction (HYPR CE). Magn Reson Med 2011;65:536-63

13. Ganguly A, Fieselmann A, Marks M, et al. Cerebral CT perfusion using an interventional C-arm imaging system: cerebral blood flow measurements. AJNR Am J Neuroradiol 2011;32: 1525-31

14. Geleijns J, Salvado AM, de Bruin PW, et al. Computed tomography dose assessment for a $\mathbf{1 6 0} \mathrm{mm}$ wide, 320 detector row, cone beam CT scanner. Phys Med Biol 2009;54:3141-59 